\title{
An Introduction to Independent Component Analysis: InfoMax and FastICA algorithms
}

\author{
Dominic Langlois, Sylvain Chartier, and Dominique Gosselin \\ University of Ottawa
}

This paper presents an introduction to independent component analysis (ICA). Unlike principal component analysis, which is based on the assumptions of uncorrelatedness and normality, ICA is rooted in the assumption of statistical independence. Foundations and basic knowledge necessary to understand the technique are provided hereafter. Also included is a short tutorial illustrating the implementation of two ICA algorithms (FastICA and InfoMax) with the use of the Mathematica software.

Nowadays, performing statistical analysis is only a few clicks away. However, before anyone carries out the desired analysis, some assumptions must be met. Of all the assumptions required, one of the most frequently encountered is about the normality of the distribution (Gaussianity). However, there are many situations in which Gaussianity does not hold. Human speech (amplitude by time), electrical signals from different brain areas and natural images are all examples not normally distributed. The well-known "cocktail party effect" illustrates this concept well. Let us imagine two people standing in a room and speaking simultaneously. If two microphones are placed in two different places in the room, they will each record a particular linear combination of the two voices. Using only the recordings, would it then be possible to identify the voice of each speaker (Figure 1a)? If Gaussianity was assumed, we could perform a Principal Component Analysis (PCA) or a Factorial Analysis (FA). The resulting components would be two new orderly voice combinations (Figure 1a). Therefore, such a technique fails to isolate each speaker's voice.

On the other hand, if non-Gaussianity is assumed, then

We wish to thank Marianna Gati for her valuable comments and helpful suggestions. This work was supported by scholarships from the Fonds québécois de recherche sur la nature et les technologies (FQRNT) and the Ontario Graduate Scholarship Program (OGS).
Independent Component Analysis (ICA) could be applied to the same problem and the result would be quite different. ICA is able to distinguish the voice of each speaker from the linear combination of their voices (Figure 1b). This reasoning can be applied to many biological recording involving multiple source signals (e.g. EEG). However, the readers must bear in mind that there are two main differences in the interpretation of extracted components using ICA instead of PCA. First, in ICA, there is no order of magnitude associated with each component. In other words, there is no better or worst components (unless the user decides to order them following his own criteria). Second, the extracted components are invariant to the sign of the sources. For example, in image processing, a white letter on a black background is the same as a black letter on a white background.

The remainder of the paper is comprised of a first section that briefly exposes the theoretical foundations of ICA $^{1}$, and of a second section that gives an example of its application using two different implemented algorithms (supplemental material). The second section also presents a short discussion on future tracks of research.

\section{Theoretical foundations of ICA}

Let us denote the random observed vector $\mathbf{X}=\left[X_{1}, X_{2}, \ldots, X_{m}\right]^{\mathbf{T}}$ whose $m$ elements are mixtures of $m$ independent elements of a random vector $\mathbf{S}=\left[S_{1}, S_{2}, \ldots, S_{m}\right]^{\mathbf{T}}$ given by

$$
\mathbf{X}=\mathbf{A S}
$$


Figure 1. Comparison between PCA (a) and ICA (b) in the context of the "cocktail party effect".

a)

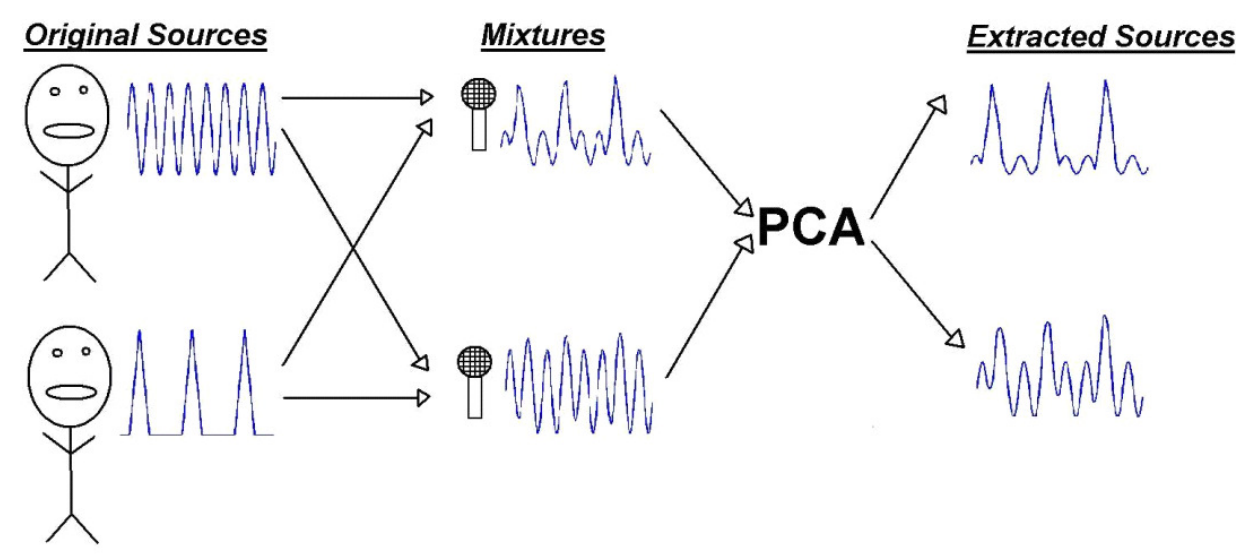

b)

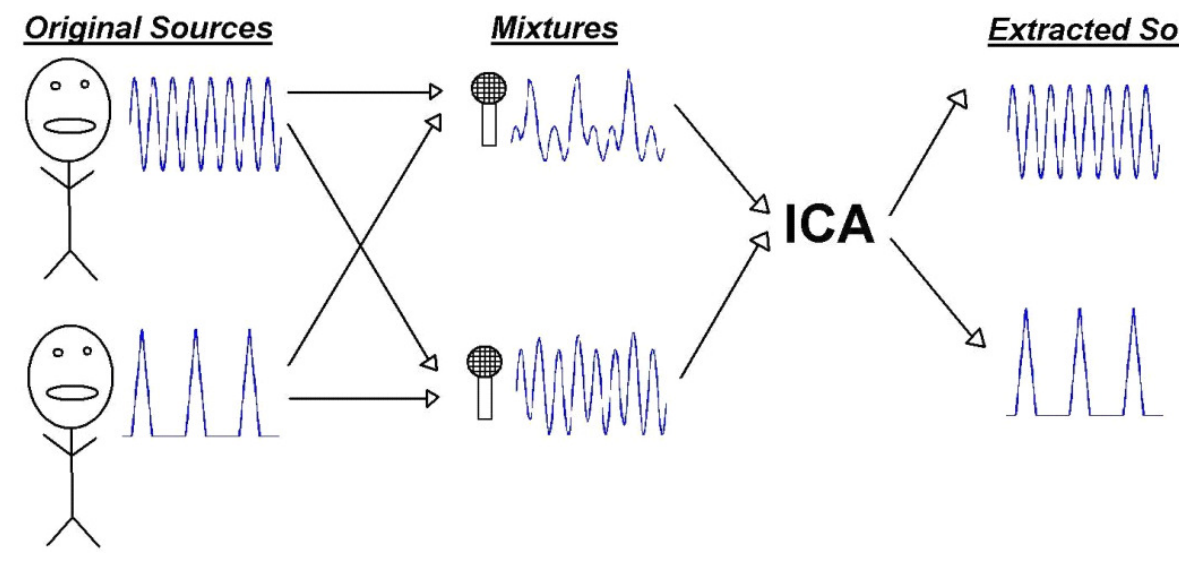

Where A represents an $m \times m$ mixing matrix, the sample value of $X_{j}$ is denoted by $x_{j}$ and $j=1,2, \ldots, m$. The goal of ICA is to find the unmixing matrix $\mathbf{W}$ (i.e. the inverse of $\mathbf{A}$ ) that will give $\mathbf{Y}$, the best possible approximation of $\mathbf{S}$ :

$$
\mathbf{Y}=\mathbf{W X} \cong \mathbf{S}
$$

In order to use ICA, five assumptions must be met. First, statistical independence between each of the sources $S_{i}$ from the sources vector $\mathbf{S}$ is assumed (independence is at the core of ICA and will be discussed further in the next subsection). Second, the mixing matrix must be square and full rank. In other words, the number of mixtures must be equal to the number of sources and the mixtures must be linearly independent from each other. ${ }^{2}$ Third, the only source of stochasticity in the model is the source vector $\mathbf{S}$ (i.e. there is no external noise). The model must thus be noise free. Fourth, it is assumed that the data are centered (zero mean).
Also, for some algorithms, the data must be pre-processed further; sometimes, the observation vector $\mathbf{X}$ must be whitened. ${ }^{3}$ Fifth, the source signals must not have a Gaussian probability density function (pdf) except for one single source that can be Gaussian.

\section{Statistical independence}

Let $x_{1}, x_{2}, \ldots, x_{m}$ be random variables with pdf $f\left(x_{1}, x_{2}, \ldots, x_{m}\right)$, then the variables $x_{i}$ are mutually independent if:

$$
f\left(x_{1}, x_{2}, \ldots, x_{m}\right)=f_{1}\left(x_{1}\right) f_{2}\left(x_{2}\right) \ldots f_{m}\left(x_{m}\right)
$$

that is, if the pdf of the $x_{i}$ is equal to the multiplication of each marginal pdf of the $x_{i}$. Statistical independence is a more severe criterion than uncorrelatedness between two variables. If we take random centered variables, uncorrelatedness is expressed by the following equation: 


$$
E\left[x_{i} x_{j}\right]-E\left[x_{i}\right] E\left[x_{j}\right]=0 \quad \text { for } i \neq j
$$

where $E[$.$] is the expectation. On the other hand,$ independence can be defined using the expectation by the following:

$$
E\left[g_{1}\left(x_{i}\right) g_{2}\left(x_{j}\right)\right]-E\left[g_{1}\left(x_{i}\right)\right] E\left[g_{2}\left(x_{j}\right)\right]=0 \quad \text { for } i \neq j_{(5)}
$$

for all functions $g_{1}$ and $g_{2}$. In the particular case where the joint pdf of the variables is Gaussian, uncorrelatedness is equivalent to independence (Hyvärinen, Karhunen \& Oja, 2000, 2001).

There are several ways to measure independence and each of them involves the use of different algorithms when it comes to performing an ICA, which results in slightly different unmixing matrices. There are two main families of ICA algorithms (Haykin, 2009). While some algorithms are rooted in the minimization of mutual information, others take root in the maximization of non-Gaussianity.

\section{Minimization of mutual information}

Mutual information is defined for a pair of random variables as:

$$
I(X ; Y)=H(X)-H(X \mid Y)
$$

where $H(X \mid Y)$ is the conditional entropy (the entropy of $X$ conditional on $\mathrm{Y}$ taking a certain value $y)$ and $H(X)$ is the entropy of $X$. Conditional entropy is given by:

$$
H(X \mid Y)=H(X, Y)-H(Y)
$$

where $H(X, Y)$ is the joint entropy of $X$ and $Y$ and $H(Y)$ is the entropy of $Y$. Formally, entropy for a given variable is defined by Shannon (1948) as:

$$
\begin{gathered}
H(X)=-\sum_{x} P(x) \log P(x) \\
H(Y)=-\sum_{y} P(y) \log P(y) \\
H(X, Y)=-\sum_{x, y} P(x, y) \log P(x, y)
\end{gathered}
$$

where $P(x)$ is the probability that $X$ is in the state $x$. Entropy can be seen as a measure of uncertainty. The lower the value the more information we have about a given system. Therefore, going back to Equation 6, mutual information can be seen as the reduction of uncertainty regarding variable $X$ after the observation of $Y$. Therefore by having an algorithm that seeks to minimize mutual information, we are searching for components (latent variables) that are maximally independent. Examples of algorithms that use minimization of mutual information can be found in Amari, Cichocki \& Yang (1996); Bell \& Sejnowski (1995a); Cardoso (1997); Pham, Garrat \& Jutten (1992).

Using Equation 6 and after some manipulation, Amari et al. (1996) proposed the following algorithm to compute the unmixing matrix $\mathbf{W}$ (called InfoMax):

1. Initialize $\mathbf{W}(0)$ (e.g. random)

2. $\mathbf{W}(t+1)=\mathbf{W}(t)+\eta(t)\left(\mathbf{I}-f(\mathbf{Y}) \mathbf{Y}^{T}\right) \mathbf{W}(t)$
3. If not converged, go back to step 2 .

where $t$ represents a given approximation step, $\eta(t)$ a general function that specifies the size of the steps for the unmixing matrix updates (usually an exponential function or a constant), $f(\mathbf{Y})$ a nonlinear function usually chosen according to the type of distribution (super or subGaussian), I the identity matrix of dimensions $m \times m$ and T the transpose operator. In the case of super-Gaussian distributions, $f(\mathbf{Y})$ is usually set to:

$$
f(\mathbf{Y})=\operatorname{Tanh}(\mathbf{Y})
$$

and for sub-Gaussian distributions, $f(\mathbf{Y})$ is set to:

$$
f(\mathbf{Y})=\mathbf{Y}-\operatorname{Tanh}(\mathbf{Y})
$$

The package InfoMax.nb is an implementation of this algorithm.

\section{Maximization of non-Gaussianity}

Another way to estimate the independent components is by focusing on non-Gaussianity. Since it is assumed that each underlying source is not normally distributed, one way to extract the components is by forcing each of them to be as far from the normal distribution as possible. Negentropy can be used to estimate non-Gaussianity. In short, negentropy is a measure of distance from normality defined by:

$$
N(\mathbf{X})=H\left(\mathbf{X}_{\text {Gaussian }}\right)-H(\mathbf{X})
$$

where $\mathbf{X}$ is a random vector known to be non-Gaussian, $H(\mathbf{X})$ is the entropy (see Equation $8 \mathrm{a})$, and $H\left(\mathbf{X}_{\text {Gaussian }}\right)$ is the entropy of a Gaussian random vector whose covariance matrix is equal to that of $\mathbf{X}$. For a given covariance matrix, the distribution that has the highest entropy is the Gaussian distribution. Negentropy is thus a strictly positive measure of non-Gaussianity. However, it is difficult to compute negentropy using Equation 11, which is why approximations are used. For example, Hyvärinen \& Oja (2000) have proposed the following approximation:

$$
N(V)=E(\phi(V))-E(\phi(U))^{2}
$$

where $V$ is a standardized non-Gaussian random variable (zero mean and unit variance), $U$ a standardized Gaussian random variable and $\phi($.$) a non-quadratic function (usually$ Tanh(.)). After some manipulation, they proposed the following algorithm (named FastICA):

1. Initialize $\mathbf{w}_{\mathrm{i}}$ (e.g. random)

2. $\mathbf{w}_{\mathbf{i}}^{+}=E\left(\phi^{\prime}\left(\mathbf{w}_{\mathbf{i}}^{\mathbf{T}} \mathbf{X}\right)\right) \mathbf{w}_{\mathbf{i}}-E\left(\mathbf{x} \phi\left(\mathbf{w}_{\mathbf{i}}^{\mathbf{T}} \mathbf{X}\right)\right)$

3. $\mathbf{w}_{\mathbf{i}}=\frac{\mathbf{w}_{\mathbf{i}}^{+}}{\left\|\mathbf{w}_{\mathbf{i}}^{+}\right\|}$

4. For $\mathrm{i}=1$, go to step 7 . Else, continue with step 5 .

5. $\mathbf{w}_{\mathbf{i}}^{+}=\mathbf{w}_{\mathbf{i}}-\sum_{\mathbf{j}=\mathbf{1}}^{\mathbf{i}-\mathbf{1}} \mathbf{w}_{\mathbf{i}}^{\mathbf{T}} \mathbf{w}_{\mathbf{j}} \mathbf{w}_{\mathbf{j}}$ 
6. $\mathbf{w}_{\mathbf{i}}=\frac{\mathrm{w}_{\mathbf{i}}^{+}}{\left\|\mathrm{w}_{\mathbf{i}}^{+}\right\|}$

7. If not converged, go back to step 2. Else go back to step 1 with $\mathrm{i}=\mathrm{i}+1$ until all components are extracted.

where $\mathbf{w}_{\mathbf{i}}$ is a column-vector of the unmixing matrix $\mathbf{W}, \mathbf{w}_{\mathbf{i}}^{+}$ is a temporary variable used to calculate $\mathbf{W}_{\mathbf{i}}$ (it is the new $\mathbf{W}_{\mathbf{i}}$ before normalization), $\phi^{\prime}($.$) is the derivative of \phi($.$) and E($. is the expected value (mean). Once a given $\mathbf{w}_{\mathbf{i}}$ has converged, the next one ( $\mathbf{w} \mathbf{i + 1})$ must be made orthogonal to it (and all those previously extracted) with Equations $13 \mathrm{c}$ and $13 \mathrm{~d}$ in order for the new component to be different from it (them). This algorithm has been implemented in the package FastICA.nb.

\section{How to use the ICA packages}

This section provides a quick overview of the InfoMax ICA package based on the maximum information perspective (InfoMax.nb; Amari et al., 1996), and on the FastICA package, based on the non-Gausianity perspective (FastICA.nb; Hyvärinen \& Oja, 2000). Both packages have been implemented using Mathematica 7.0 and contain the same options with the exception of some parameters that are unique to a given algorithm. Each package consists of two main sections: Functions and Main. The Functions section contains the implementation of the algorithm and the necessary accompanying auxiliary functions. This section must be activated before ICA can be performed using the Main section. The Main section is divided into three cells: the information about the various parameters that need to be set prior to the analyses.

\section{Parameters}

First, the type of data must be specified in order to display the results properly (Figure 2). For example, if the data are in a sound file format (.wav), type must equal "sound" and sampleRate must be set to the correct desired frequency for the software to play the file correctly. Setting type to "image" allows for the use of usual image file formats (e.g., .jpg and .bmp). Since the analysis is only performed on greyscale images, any colour images will be automatically converted. If the data are time series, but not sound, then type must be set to "temporal" and a graph will depict the data using time as the independent variable. Finally, setting type to "other" is used for any data in a text format (e.g., .dat or .txt) (Each mix must be in a different file and arranged in a column). The next two options are about the convergence of the algorithm. First, minDeltaW controls the minimum difference between a given approximation $\mathbf{W}(t)$ and the next one $\mathbf{W}(t+1)$. The lower the value, the better the estimation of the source will be. However, in some cases, the algorithm may not find a proper solution and, as a precaution, maxStep will control the maximum number of allowed steps before it stops searching. Finally, for the InfoMax notebook only, the type of distribution of the sources (typeOfDist) must be given in advance for the algorithm to be able to find the correct solution. To this end, there are two possible distributions: sub-Gaussian ("Sub")

Figure 2. Screen capture featuring an example of the various parameters to be set before performing the analysis.

\section{Parameters}

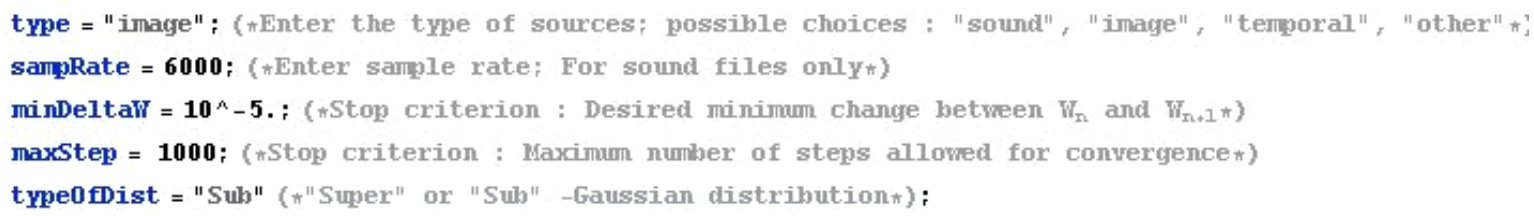

Figure 3. Example of five mixed signals to be loaded.

$\{\operatorname{mix}, \operatorname{nbCol}\}=\operatorname{mixingSign}[$ IdentityMatrix $[5]] ;$

Figure 4. Examples of "infoMaxICA" and "fastICA" functions to perform ICA.

ic = infolaxICA [mix, typeofDist, minDeltaw, maxStep, nbCol] ;

ic = fastICA [mix, minDeltaw, maxstep, nbCol] ;

parameters, sources and ICA. The Parameters cell contains

and super-Gaussian ("Super"). 
Figure 5. Syntax used to load three mixed sources (a) from a file selection window (b).

a) $\{$ mix, nbCol $\}=$ mixingSign [Identit yMatrix [3]];

b)

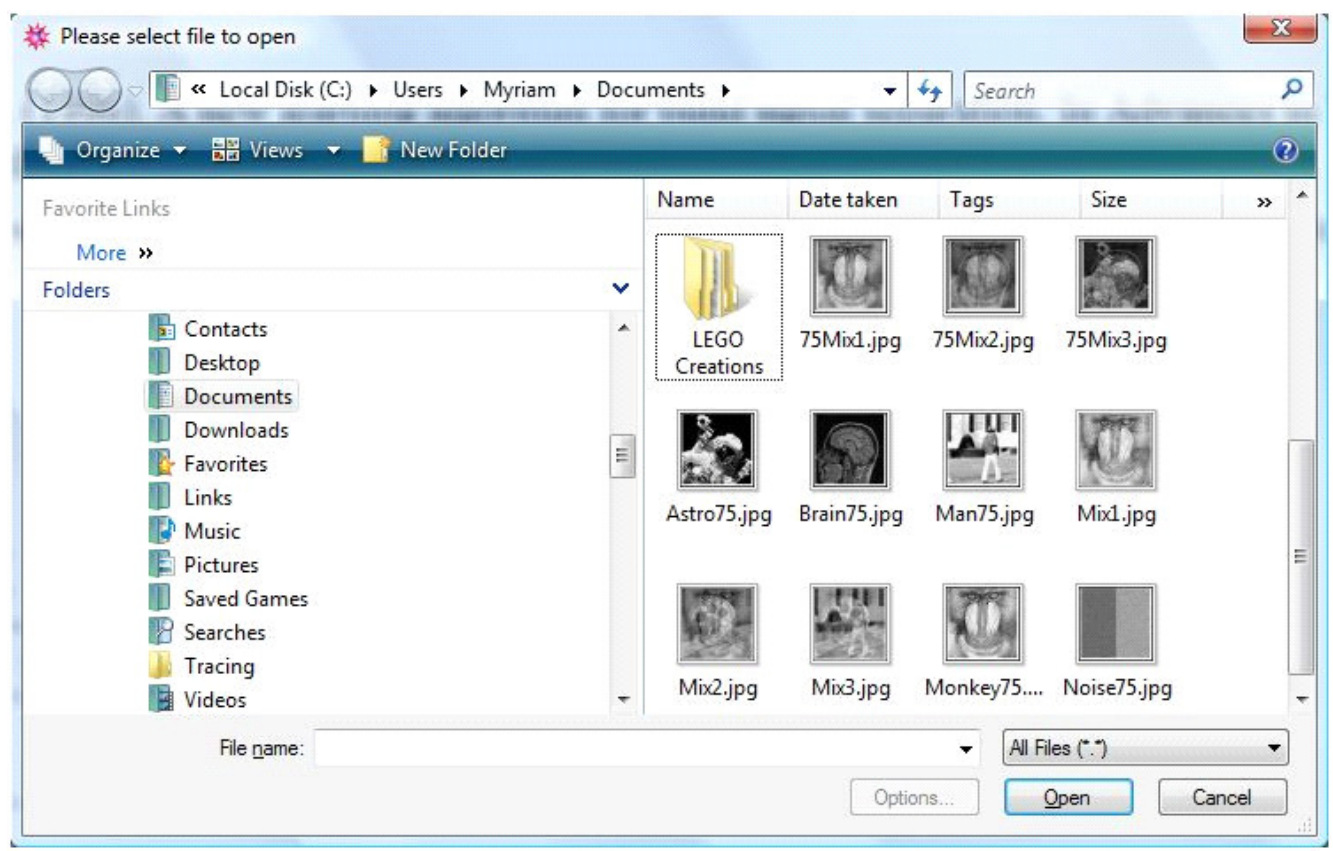

Sources

The second cell must be activated to load the mixes. Two options are offered: mixed or unmixed sources. Mixed sources are obviously the ones that are most commonly encountered. In this case, the function mixingSign[ ] will need IdentiyMatrix $[m]$ as an argument; where $m$ is the number of sources (Figure 3).

If the sources are not mixes (e.g. to use the packages for illustration purposes), then the notebook will generate a random mixing matrix or alternatively the user can provide one. Finally, once activated, a window will appear requesting the location of each file. Once loaded, the sources will be displayed accompanied by correlation matrices.

\section{Performing ICA}

Finally, to perform the ICA, the function infoMaxICA[ ] or fastICA[ ] must be activated (Figure 4). Once the analysis is completed, the notebook will display the extracted sources as well as the correlation matrix of the extracted sources.

\section{Example}

In this example, Infomax and FastICA algorithms are used to extract the components from three mixes of images (provided in the supplemental materials). Also, for comparison, Principal Component Analysis (PCA) will be performed on the same mixes.

\section{Infomax and FastICA}

After the "Functions" section has been activated, the parameters were set as follows:

- type = "image"

- sampRate non-applicable in this case

- minDeltaW $=10^{\wedge}-5$

- maxStep = 2500 for Infomax

- maxStep = 100 for FastICA (i.e. 100 for each component)

- For InfoMax, typeOfDist = "Sub" and "Super". Since no information about the underlying distribution was available, both types were tried.

Once the parameters are set, three "image" mixed sources were loaded. To that end, IdentityMatrix[3] was used as an argument for the function mixingSign[ ] (Figure 5).

Once the images are loaded, the notebook illustrates the loaded data (Figure 6). In this example, since the signals are already mixes, both the original and mixed signals are the same.

The ICA is then performed (Figure 7). The output of the 
analysis shows the extracted components (in this case, images) and the correlation matrix of those components.

Since ICA is invariant to the sign of the sources, extracted components are illustrated using the two possible signs (background). Finally, a correlation matrix accompanies the outputs to verify that they are not correlated.

PCA vs Infomax vs FastICA

The same mixes were used to compare PCA, InfoMax super (typeOfDist set to super-Gaussian), InfoMax sub (typeOfDist set to sub-Gaussian), and FastICA. As expected, PCA and one of the InfoMax analyses (Infomax super) were unable to find the independent components, since the source signals used in the example are sub-Gaussian. On the other hand, InfoMax sub and FastICA performed particularly well (Figure 8).

\section{Discussion}

Readers are encourages to use special softwares that allow various situations to be taken into account. For example, FastICA implementations in Matlab, $\mathrm{C}++, \mathrm{R}$ and
Python can be accessed through the Laboratory of Computer and Information Science: Adaptive Informatics Research Center website (http://www.cis.hut.fi/projects/ica/fastica/). There are also many practical considerations that must be taken into account that goes beyond the scope of this paper. For example, it is common practice to pre-whiten the data, which was done for the FastICA notebook.

Furthermore, many theoretical links can be made between the different ICA algorithms. For examples, algorithms that minimize mutual information are linked together whether they use the Kullback-Leibler divergence (Amari et al., 1996), maximum likelihood (Pham et al., 1992) or maximum entropy (Bell \& Sejnowski, 1995a; Cardoso, 1997) to do so. Usually, to perform ICA and other blind source separation problems, five conditions must be met: 1 The source signals must be statistically independent; 2 - The number of source signals must equal the number of mixed observed signals and mixtures must be linearly independent from each other; 3 - The model must be noise free; 4 - Data must be centered and; 5 - The source signals must not have a Gaussian pdf, except for one single source that can be Gaussian.

Figure 6. Original signals, mixed signals and mixes correlation matrix for the loaded data.

Original Signals
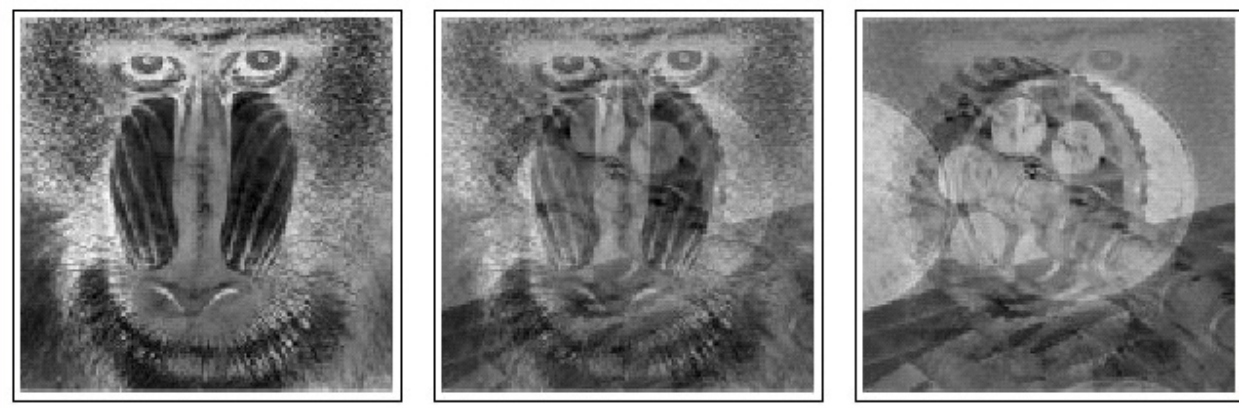

Mixed Signals
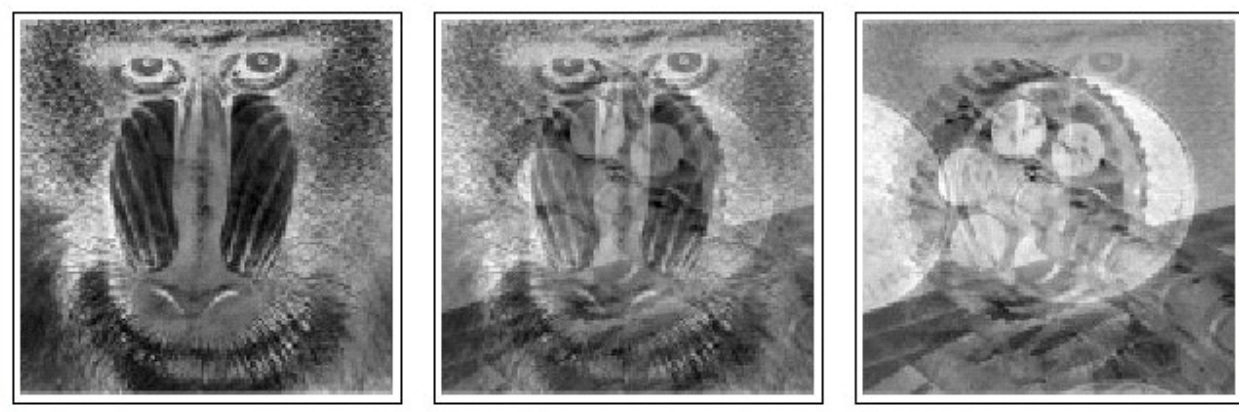

\begin{tabular}{l|lll} 
& Mix 1 & Mix 2 & Mix 3 \\
\hline Mix 1 & 1. & 0.850107 & 0.362291 \\
Mix 2 & 0.850107 & 1. & 0.77725
\end{tabular}

\begin{tabular}{l|lll}
$\operatorname{Mix} 3$ & 0.362291 & 0.77725 & 1.
\end{tabular} 
Figure 7. Outputs of the ICA (FastICA).
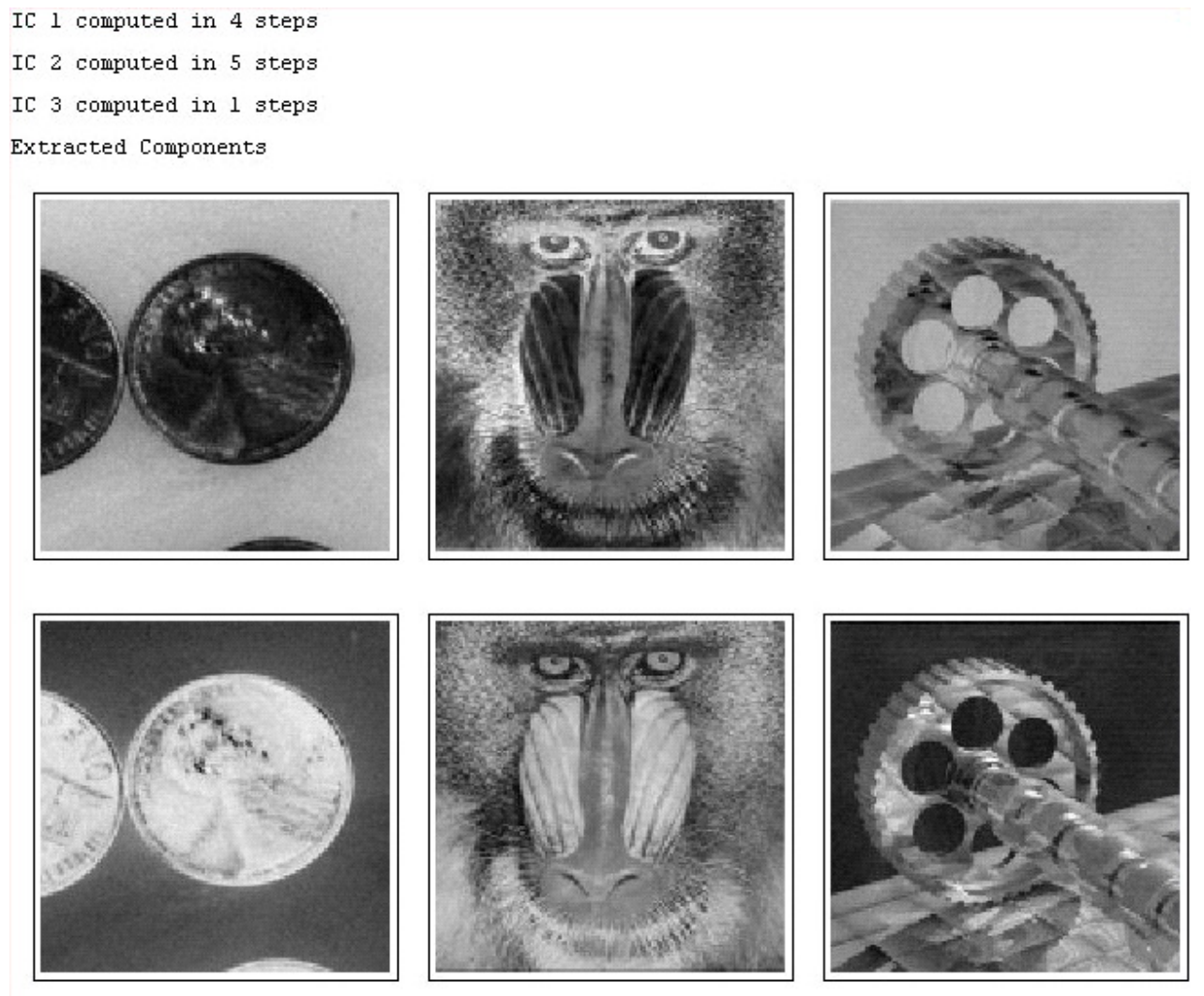

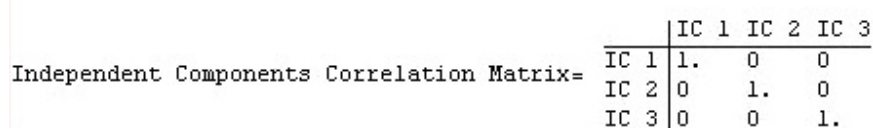

The main advantages of algorithms based on the minimization of mutual information are their ability to adapt to variations in the environment and the fact that they are robust if the right type of distribution is provided (super- or sub-Gaussian). On the other hand, algorithms based on negentropy, e.g. FastICA, also have interesting features (Haykin, 2009). FasICA is able to find a solution quickly and is robust to the type of distribution (Haykin, 2009). ICA is presently an expanding field and many interesting possibilities are currently on our doorstep. Such possibilities include ICA for nonlinear mixing process, ICA for source signals that are noisy, ICA for a number of source signals greater than the number of observables (like our brain does with only two ears!) and blind source separation techniques based on temporal dependencies (Haykin, 2009). In short, ICA is a technique that will be impossible to avoid in a near future for any researcher involved in source signals extraction.

\section{References}

Amari, S., Cichocki, A., \& Yang, H. H. (1996). A new learning algorithm for blind signal separation. Advances in Neural Information Processing Systems, 8, 757-763.

Bell, A. J., \& Sejnowski, T. J. (1995a). A non-linear information maximization algorithm that performs blind separation. Advances in Neural Information Processing Systems, 7, 467-474.

Bell, A. J., \& Sejnowski, T. J. (1995b). An informationmaximization approach to blind separation and blind deconvolution. Neural Computation, 7, 1129-1159.

Cardoso, J.-F. (1997). Infomax and maximum likelihood for source separation. IEEE Letters on Signal Processing, 4, 112-114.

Comon, P. (1994) Independent component analysis, a new concept?. Signal Processing, 36, 287-314.

Haykin, S. (2009). Neural Networks and Learning Machines. New Jersey : Pearson Education, Inc.

Hérault, J., \& Ans, B. (1984). Circuits neuronaux à synapses modifiables: décodage de messages composites par apprentissage non supervisé. C.-R. de l'Académie des Sciences, 299(III-133), 525-528.

Hérault, J., Jutten, C., \& Ans, B. (1985). Détection de grandeurs primitives dans un message composite par une architecture de calcul neuromimétique en 
Figure 8. Outputs from PCA, ICA (InfoMax super), ICA (InfoMax sub) and FastICA.

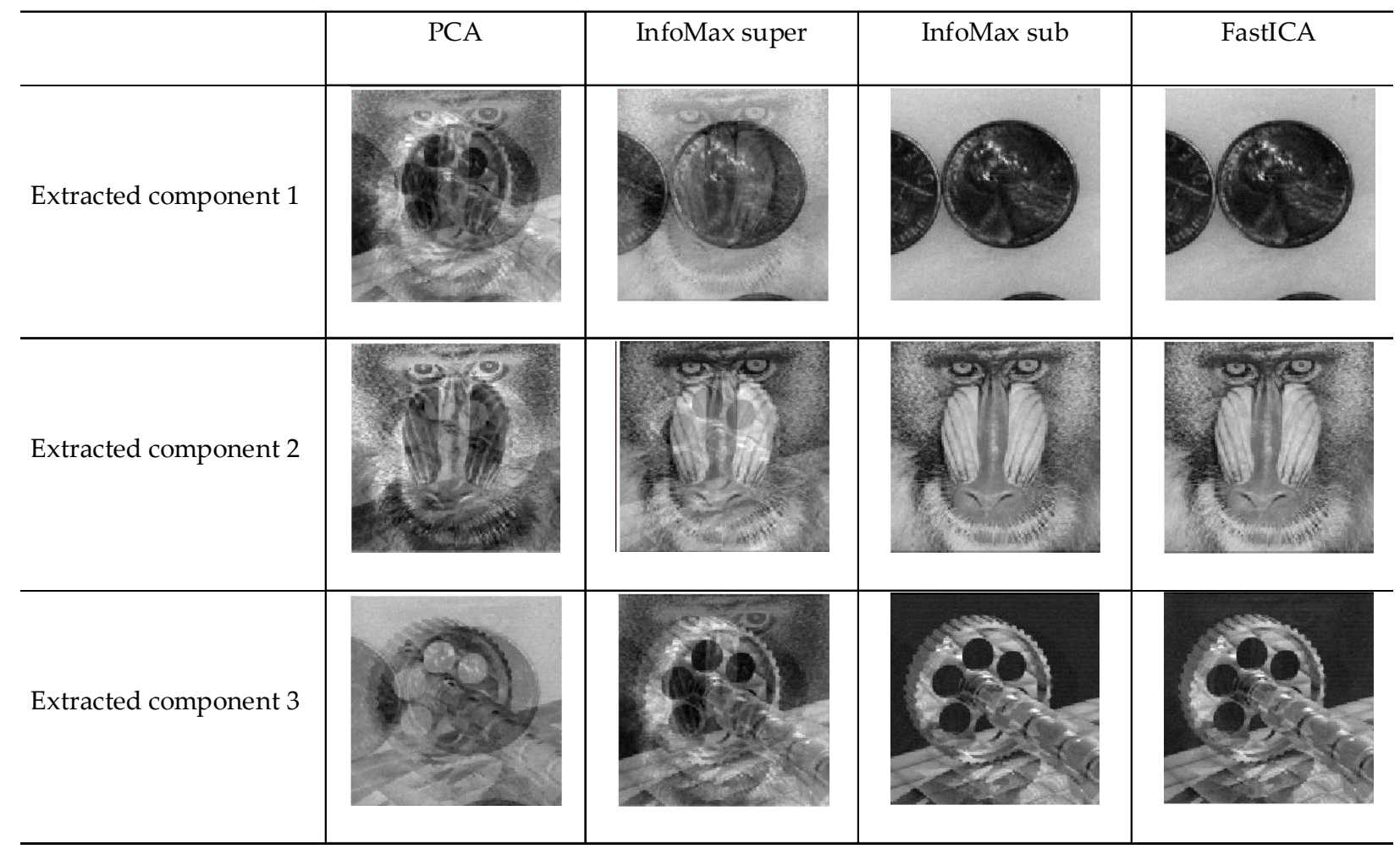

apprentissage non supervisé. Actes du Xème colloque GRETSI, 1017-1022.

Hyvärinen, A. (1998). New approximations of differential entropy for independent component analysis and projection pursuit. Advances in Neural Information Processing Systems, 10, 273-279.

Hyvärinen, A. (1999a). Survey on Independent Component Analysis. Neural Computing Surveys, 2, 94-128.

Hyvärinen, A. (1999b). Fast and robust fixed-point algorithms for independent component analysis. IEEE Trans. On Neural Networks, 10, 626-634.

${ }^{1}$ Independent component analysis (ICA) was introduced in the 80 s by J. Hérault, C. Jutten and B. Ans, (Hérault \& Ans, 1984; Hérault, Jutten, \& Ans, 1985) in the context of studies on a simplified model of the encoding of movement in muscle contraction. During that decade, ICA remained mostly unknown at the international level. In 1994, the name "independent component analysis" appeared for the first time in the paper "Independent component analysis, a new concept?" written by Comon. The technique finally received attention from a wider portion of the scientific community with the publication of an ICA algorithm based on the InfoMax principle (Bell \& Sejnowski, 1995a, 1995b). Since
Hyvärinen, A., Karhunen, J., \& Oja, E. (2001). Independent Component Analysis. New York: John Wiley \& Sons, inc.

Hyvärinen, A., \& Oja, E. (2000). Independent component analysis: algorithms and applications. Neural Networks, 13, 411-430.

Pham, D.-T., Garrat, P. \& Jutten, C. (1992). Separation of a mixture of independent sources through a maximum likelihood approach. Proceedings EUSIPCO, 771-774.

\section{Manuscript received November 28th 2009 \\ Manuscript accepted March 30th 2010.}

then, ICA has become a well establish area of research in which many papers, conferences and seminars are now commonly available.

${ }^{2}$ However, this requirement can be relaxed (see for example Hyvärinen \& Oja, 2000).

${ }^{3}$ The observation vector must be linearly transformed so that the correlation matrix gives: $E\left[\mathbf{X X}^{\mathbf{T}}\right]=\mathbf{I}$. 